\title{
On the onset of chaotic motion in deterministic systems
}

\author{
Ramakrishna Ramaswamy and R. A. Marcus \\ Arthur Amos Noyes Laboratory of Chemical Physics, ${ }^{\text {a) }}$ California Institute of Technology, Pasadena, \\ California 91125 \\ (Received 16 June 1980; accepted 26 September 1980)
}

In the present paper the classical counterpart of the quantum avoided crossing method for detecting chaos is described using classical (Lie-transform) perturbation theory and a grid of action variables. The results are applied to two systems of coupled oscillators with cubic and quartic nonlinearities. The plots of energy of members of the grid versus the perturbation parameter provide a visual description for predicting the onset of chaos.

\section{INTRODUCTION}

The study of classical dynamical systems ${ }^{1}$ is currently of great interest, partly because analogous problems (regular versus irregular motion) arise in numerous other fields. ${ }^{2}$ In the present paper, a method for studying the onset of chaotic motion in classical systems is presented. Chaos is of particular interest in the behavior of isolated molecules, since theories of unimolecular reactions, chemical activation and infrared multiphoton dissociation frequently assume its presence (e.g., RRKM for unimolecular reactions) or absence (e.g., N. B. Slater's theory).

For a system with $N$ degrees of freedom, i.e., $N$ coordinates $q_{1}, \ldots, q_{N}$ and the conjugate momenta $p_{1}, \ldots$, $p_{N}$, the Hamiltonian function is $\mathscr{H C}\left(q_{1} \ldots q_{N}, p_{1} \ldots p_{N}\right)$. Such a system is termed integrable ${ }^{1}$ if there exist $N$ independent smooth functions of q, p, the "isolating integrals," $f_{1}, \ldots, f_{N}=\mathcal{H}$ (the total energy) with vanishing Poisson brackets $\left\{f_{i}, f_{j}\right\}=0$ for all $i, j$. (This is trivially true for $N=1$, and so, in the rest of the discussion $N \geq 2$.) It can be shown that integrability implies $^{1}$ the existence of a suitable system of canonical coordinates, the so-called action-angle variables. In this case, the dynamical flow of the Hamiltonian in the $2 N$-dimensional phase space actually occurs on subspaces of dimension $N$, on which the $N$ action variables are constant. The motion can then be described as a Fourier series in at most $N$ fundamental frequencies, their overtones, and combinations. ${ }^{1}$

For nonintegrable systems one might imagine that the only isolating integral is the total energy (and for field-free systems in three dimensions, the total angular momentum and any space fixed component), thus leading to ergodic flow on the energy hypersurface. ${ }^{3}$ In the typical case, however, the flow of a dynamical system is neither integrable nor ergodic. ${ }^{4}$

For a large class of nonlinear Hamiltonian systems, the theorem of Kolmogorov-Arnol'd and Moser (KAM) ${ }^{1,5}$ assures the existence of stable (regular) motion on $N$-dimensional submanifolds in the $2 N$-dimensional phase space, for sufficiently small perturbations. Unfortunately the theorem guarantees the existence of such motion without any reasonable estimate of how much (as a measure) of the flow is close to the integrable

\footnotetext{
${ }^{\text {a) }}$ Contribution No, 6240
}

flow or of how large the perturbation must be to give rise to any appreciable amount of such irregular behavior.

Numerical experiments have provided evidence of KAM type behavior ${ }^{6}$ for many typical nonintegrable systems as for example in the early work of Hénon and Heiles, ${ }^{7}$ where the Hamiltonian

$$
\mathcal{H C}=\frac{1}{2}\left(p_{1}^{2}+p_{2}^{2}+q_{1}^{2}+q_{2}^{2}\right)+q_{1}\left(q_{2}^{2}-\frac{1}{3} q_{1}^{2}\right)
$$

was studied over a range of energies. (The variation of energy is equivalent, via a scaling, to variation in the strength of the nonlinear perturbation.) At low energies, the flow was seen to be almost entirely quasiperiodic (flow on a 2-dimensional torus), being a small perturbation of the behavior of the integrable system,

$$
H_{I}=\frac{1}{2}\left(p_{1}^{2}+p_{2}^{2}+q_{1}^{2}+q_{2}^{2}\right) .
$$

At high energies, an appreciable fraction of the classical trajectories was observed to be no longer regular (quasiperiodic), corresponding to the lack of existence of (local) action-angle coordinates, or equivalently, due to the absence of tori. In current terminology, this latter type of motion is called "cha otic." The differences in the two types of behavior is easily visualized in several ways: The Poincare surfaces of section ${ }^{7}$ for regular motions are smooth closed curves while for chaotic motions they are an apparently random splatter of points; the spectrum of the motion ${ }^{8}$ (Fourier transform of the coordinates) is a discrete set of lines for the regular case and a "grassy" broad set of lines for the chaotic case; the rate of separation of nearby regular trajectories is linear in time, whereas for chaotic motions, it is exponential. ${ }^{6,9}$

The energy range over which the motion changes character, from being primarily regular to primarily chaotic, is usually quite small; this has become known as the "stochastic" or "chaotic" transition. Predicting this transition for arbitrary systems is no simple task ${ }^{10}$ and a variety of methods have been proposed. ${ }^{11}$ In the present paper we describe a method for studying and predicting this onset for systems that are perturbed oscillators with no low zeroth order internal resonances. We consider, in particular, when, as a function of the energy and where in phase space, local chaos is likely to occur.

The remainder of the paper is organized as follows. 
In Sec. II, the proposed method is outlined and the connection with recent quantum-mechanical studies ${ }^{12,13}$ on the role of multiple "avoided crossings" in producing quantum chaos is described. In Sec. III, the method is applied to two simple examples of two coupled oscillators with cubic and quartic nonlinearities. Finally, in Sec. IV, the summary is given.

\section{USE OF AN ACTION LATTICE}

We consider a particular class of conservative Hamiltonians of the general form

$$
\mathfrak{H}=\frac{1}{2} \sum_{i=1}^{N}\left(p_{i}^{2}+\omega_{i}^{2} q_{i}^{2}\right)+\lambda\left\{\sum_{i, j, k}^{N} \alpha_{i j k} q_{i} q_{j} q_{k}\right\} \ldots,
$$

where $N \geq 2$ and the p's and q's are canonically conjugate variables such that

$$
\dot{q}=\frac{\partial \mathcal{H}}{\partial p}, \quad-\dot{p}=\frac{\partial \mathcal{H}}{\partial q},
$$

and the zeroth order frequencies, $\omega$ do not satisfy any "dangerous" commensurabilities (in the sense of secular perturbations). For such Hamiltonians, a successive series of transformations can be performed (via standard classical canonical perturbation methods ${ }^{14}$ ), to convert $\mathfrak{F}$ to a function of new variables, $\xi$, $\eta$, such that

$$
\mathcal{H C}(\mathbf{p}, q ; \lambda) \rightarrow F(\xi, \eta ; \lambda),
$$

with

$$
\xi=\partial F / \partial \eta, \quad \eta=-\partial F / \partial \xi,
$$

and

$$
F(\xi, \eta ; \lambda)=f\left(\xi^{2}+\eta^{2} \omega^{-2} ; \lambda\right),
$$

i.e., the transformed Hamiltonian is a function of the new variables $\xi$, $\eta$, only in the combination $\left(\xi^{2}+\eta^{2} \omega^{-2}\right)$. This is merely the Birkhoff normal form ${ }^{15}$ of $\mathcal{H}$, and is a power-series expansion in $\lambda$. For the present, we are not concerned with the convergence properties of the normal form ${ }^{14,15}$; in practice, a low order expansion in $\lambda$ has sufficed in the systems we have studied for providing a more than adequate description of $\mathfrak{K}$ for the purpose at hand. The transformation to good action-angle variables, I, $\theta$ for $\mathcal{H}$ is effected by

$$
\begin{aligned}
& \xi=\sqrt{I / \pi \omega} \cos 2 \pi \theta, \\
& \eta=\sqrt{I \omega / \pi} \sin 2 \pi \theta,
\end{aligned}
$$

such that

$$
F(\boldsymbol{\xi}, \eta ; \lambda) \rightarrow K(\mathbf{I} ; \lambda)
$$

and $K$ is a function of the new action variables $I$ alone. For $\lambda=0$, the transformations are exact; the question which we now consider is; What can be learned about the behavior of the true Hamiltonian $\mathcal{H}$ by studying its (integrable) approximate $K$ ? There are some points to be noted. Firstly, $K$ is integrable (and thus can show no chaos), whereas $\mathcal{H}$ is not (and can thus exhibit chaotic motion). Secondly, since the $I$ 's are constant in $K$, i.e., $-\theta K / \partial \theta=\dot{I}=0$, fixing a set of actions I defines a torus for $F$. Some tori persist and some are destroyed by a perturbation $\lambda$. For the former, "good" action-angle variables, with actions being constants of the motion, can be defined, while for the latter they cannot, and these will be termed unstable tori.

The preceding analysis would be of no particular value if there was no stability criterion for such tori. We can, however, obtain such a criterion by the following construct. A recently proposed definition ${ }^{13}$ of quantum stochasticity is in terms of avoided crossings of energy eigenvalues with respect to the variation of a parameter. There, one examines the energy eigenstates of a Hamiltonian, $\mathfrak{H}$,

$$
\mathcal{H}=H_{0}+\lambda H_{1},
$$

where $H_{0}$ is an integrable Hamiltonian, and $H_{1}$ is a nonintegrable perturbation, by exact quantum mechanical methods, and observes the levels that avoid crossing. It was proposed ${ }^{13}$ that overlapping avoided crossings, rather than isolated ones, gave rise to a "statistical" character in the quantal eigenfunctions, and thus to apparently erratic spacings in the eigenvalue spectrum, producing thereby the irregular spectrum of Percival, ${ }^{16}$ It was further seen that the location of such avoided crossings in energy-parameter space, can be simply determined by the use of quanta $1^{12}$ perturbation theory for the system examined.

They could also be determined by semiclassical perturbation theory. Now, semiclassical quantization is essentially a procedure of constructing good actionangle variables either by trajectory ${ }^{17}$ or perturbation methods ${ }^{18}$ and then applying Einstein-Brillouin-KellerMaslov rules. ${ }^{19}$ Trajectory methods are dependent on finding invariant tori for a system with Hamiltonian $\mathscr{K}$, tori on which the action integrals along $N$ independent paths $C_{i}$ are individually quantizable, i.e.,

$$
\oint_{c_{i}} \mathrm{p} \cdot \mathrm{dq}=\left(n_{i}+\alpha_{i} / 4\right) h
$$

where $n_{i}$ is an integer, $\alpha_{i}$ the Maslov index, and $h$ the unit of action (Planck's constant). Accordingly, a quantum state then corresponds to such an invariant torus. Since it is easier to perform the semiclassical quantization by perturbation methods, the variation of semiclassical eigenvalues with a parameter is most easily studied by quantization of the normal form, i.e., by the further transformation,

$$
I \rightarrow(n+\alpha / 4) \hbar \text {, }
$$

so that

$$
K(\mathrm{I} ; \lambda) \rightarrow E(\mathrm{n} ; \alpha ; \lambda),
$$

where the $n$ 's are (integers) the good quantum numbers. For a particular choice of $n$, then, $E(n)$ is the semiclassical eigenvalue. In examining the eigenvalue vs parameter variation, the crossing (degeneracy) of more than two such eigenvalues in a small $\lambda$ neighborhood may be interpreted as the signature of overlapping avoided crossings in the true system, and thus as a signature of torus destruction, when the exact levels are "split" at the crossings.

The above statements have been phrased for a particular set of tori, namely the "quantizable" ones: 
Those with actions of integer plus $\alpha / 4$. Since classical chaos occurs through the destruction of arbitrary tori, we now seek to extend the above ideas to include all tori and so to be classical. This can be done in $N$-dimensional action space: One difficulty in achieving this objective is the fact that the classical actions are continuous variables while quantization selects only a discrete set of realizable actions; thus, the analog of curve crossings for the classical case may be difficult to visualize.

This particular obstruction is overcome in the present paper by using a discrete set of actions to examine such resonant interactions between tori. The set of actions are chosen to correspond to a "lattice" integer separations in the unit of action, denoted by $c$. Semiclassical quantum mechanics corresponds to a particular placement of this lattice on the space of actions such that one selects only those actions equal to $(\mathrm{n}+\alpha / 4) h$, i.e., $c=h$. To study the stability of all tori via the analog of curve crossing, we need merely to study the parameter dependence of sets of actions equal to $(\mathbf{n}+\alpha / 4+\chi) c$ with $\chi$ fixed for a given set, ${ }^{21(2)}$ corresponding to a particular placement of the lattice in action space. By varying $\chi^{21(b)}$ and using the above grid, one can scan the results for isolated and multiple avoided crossings. Since the energies of tori with arbitrary actions can be easily determined through $K(\mathbb{I} ; \lambda)$, this latter task reduces to the study of simple polynomial functions in the present instance. Further, since $K, F$, and $F^{\prime}$ are related by known transformations, knowing the particular tori of $F$ that are unstable is equivalent to knowing where toroidal instability occurs in the phase space for $\mathscr{K}$.

The connection of these suggestions on isolated and overlapping avoided crossings with another concept is as follows. For each crossing, there is a zero frequency $(\partial K / \mathrm{aI}=0)$ for some action $\mathrm{I}$, a linear combina tion of actions $I_{i}$, and so there is a periodic trajectory and an internal resonance. If there are two avoided crossings in the neighborhood of each other with different rotation numbers, i.e., different integers $m_{i}$ for the two resonances $\sum m_{i} \omega_{i}=0$, for each there are two periodic orbits in the vicinity of each other and two different resonances.

Chirikov has postulated ${ }^{11 b}$ that when these resonances overlap, and indeed when they become dense, one has chaos. Perturbations of nested tori normally leave the tori deformed but still nested. However, if each torus is associated with a periodic trajectory, their mutual perturbation causes the two periodic trajectories to break up into "islands" with elliptic and hyperbolic fixed points. This intersection of tori is presumed to cause local chaos. ${ }^{11 \mathrm{~b}, 22}$ A different approach with the same objective, i.e., locating periodic orbits, has been proposed by Greene. ${ }^{11(c)}$

The above discussion of the method proposed here will be illustrated using two examples with $N=2$ dimensions in the following section.

\section{TWO EXAMPLES}

\section{A. The Barbanis Hamiltonian 23}

The Hamiltonian

$$
H \mathcal{H}(\mathbf{p}, \mathbf{q} ; \lambda)=\frac{1}{2}\left(p_{1}^{2}+p_{2}^{2}+\omega_{1}^{2} q_{1}^{2}+\omega_{2}^{2} q_{2}^{2}\right)+\lambda q_{1} q_{2}^{2}
$$

has been studied extensively in the literature. ${ }^{24}$ Here we avoid the choice $\omega_{1} / \omega_{2}=1$ or 2 to within $O(\lambda)$. The canonical transformation to normal form is easily accomplished through the Lie transform, ${ }^{25}$ giving

$$
\begin{aligned}
F(\boldsymbol{\xi}, \boldsymbol{\eta} ; \lambda)= & \frac{1}{2}\left[\left(\xi_{1}^{2}+\eta_{1}^{2} / \omega_{1}^{2}\right) \omega_{1}^{2}+\left(\xi_{2}^{2}+\eta_{2}^{2} / \omega_{2}^{2}\right) \omega_{2}^{2}\right] \\
& +\frac{\lambda^{2}}{2 \omega_{1}^{2}\left(\omega_{1}^{2}-4 \omega_{2}^{2}\right)}\left[\left(\omega_{2}^{2}-3 \omega_{1}^{2} / 8\right)\left(\xi_{2}^{2}+\eta_{2}^{2} / \omega_{2}^{2}\right)^{2}\right. \\
& \left.+\omega_{1}^{2}\left(\xi_{1}^{2}+\eta_{1}^{2} / \omega_{1}^{2}\right)\left(\xi_{2}^{2}+\eta_{2}^{2} / \omega_{2}^{2}\right)\right]+O\left(\lambda^{4}\right) .
\end{aligned}
$$

The subsequent transformation to action-angle variables is direct, using

$$
I_{i}=\left(\xi_{i}^{2}+\eta_{i}^{2} / \omega_{i}^{2}\right) \omega_{i} \pi .
$$

One has

$$
\begin{aligned}
K(\mathbf{I} ; \lambda)= & \omega_{1}\left(I_{1} / 2 \pi\right)+\omega_{2}\left(I_{2} / 2 \pi\right)+\frac{\lambda^{2}}{2 \omega_{1}^{2}\left(\omega_{1}^{2}-4 \omega_{2}^{2}\right)}\left[\left(\omega_{2}^{2}-3 \omega_{1}^{2} / 8\right)\right. \\
& \left.\times I_{2}^{2} / \pi^{2} \omega_{2}^{2}+\left(\omega_{1} / \omega_{2}\right)\left(I_{1} I_{2} / \pi^{2}\right)\right]+O\left(\lambda^{4}\right) .
\end{aligned}
$$

In order to apply the method outlined in the previous section, we finally obtain the polynomial

$$
\begin{aligned}
E(\mathbf{n}, \boldsymbol{X} ; \lambda)= & \omega_{1}\left(n_{1}+\alpha_{1} / 4+\chi_{1}\right) c / 2 \pi+\omega_{2}\left(n_{2}+\alpha_{2} / 4+\chi_{2}\right) c / 2 \pi \\
& +\beta_{1}\left(n_{2}+\alpha_{2} / 4+\chi_{2}\right)^{2} c^{2} / 4 \pi^{2}+\beta_{2}\left(n_{1}+\alpha_{1} / 4+\chi_{1}\right) \\
& \times\left(n_{2}+\alpha_{2} / 4+\chi_{2}\right) c^{2} / 4 \pi^{2}+O\left(\lambda^{4}\right)
\end{aligned}
$$

The Maslov indices $\alpha_{1}$ and $\alpha_{2}$ are both equal to 2 in this case and the unit of action here is $c$. The procedure for determining the Lie transform is well known (in particular for the Barbanis Hamiltonian ${ }^{25 b}$ ) and the details will not be given here. As a straightforward application of the method, we present the curves for the set of actions with $\chi_{1}=\chi_{2}=-\frac{1}{2}$ (i.e., the lattice picks out actions exactly equal to integers) and with a unit of action $c=2 \pi$. The parameters of the Hamiltonian (3.1) are $\omega_{1}^{2}=1.6$ and $\omega_{2}^{2}=0.9$. It can be seen in Fig. 1 that the lowest multiple crossing of curves occurs at $E=19$ units in the neighborhood of $\lambda=-0.08$, the value of $\lambda$ at which the system has been studied frequently. ${ }^{24}$ This is indeed the value of the energy at which Sorbie and Handy ${ }^{24}$ report the presence of chaotic motion (we have independently confirmed this observation by examining the rate of separation of nearby trajectories at this energy ${ }^{27}$ ) by observing the Poincaré surfaces of sections of a several trajectories of the Hamiltonian (3.1).

One may obtain additional information from curves such as these. Since the actions are well defined along these curves, one can use the perturbation theory to determine the (approximate) initial conditions for the tori of the exact $\mathcal{H}$, and how they behave in the vicinity of the avoided crossings of the action set. With $\chi=-\frac{1}{4}$ this is done for some values of $\lambda$ around $\lambda=-0.08$. 
Shown in Figs. 2, 3, 4, and 5 are some energy versus $\lambda$ plots, the pertinent trajectories in $\left(q_{1}, q_{2}\right)$ space, the corresponding Poincare surfaces of section and their Fourier spectra, ${ }^{8}$ for two of these curves in Fig. 2.

These diagrams are helpful in visualizing the change that the tori undergo as a function of the perturbation. The curves in Fig. 2 are labeled $n_{1} n_{2}$, so that the actions for the respective tori are $n_{1}+\frac{1}{4}, n_{2}+\frac{1}{4}$. (Due to the symmetry of the perturbed Hamiltonian, only even separations in the $n_{2}$ 's need be considered.) Note that there are both isolated avoided crossings and multiple avoided crossings; specifically around $\lambda=-0.08$, we expect the tori with $\left(n_{1}, n_{2}\right)$ equal to $(2,18)$ and $(5,14)$ to display the most sensitivity to perturbation. The topmost curve, $\mathbf{n}=(11,6)$ in Fig. 2, undergoes no crossings when $\lambda=-0.08 \pm 0.01$, and thus we expect the torus to be stable in that $\lambda$ interval even though the energy is very close to the dissociation energy.

In Fig. 3 , the trajectories of the $\left(n_{1}, n_{2}\right)$ tori, ${ }^{28}(11,6)$ and $(2,18)$ are shown; as expected, the former is stable at all values of $\lambda$ in Fig. 3, while the latter, which has undergone a multiple avoided crossing around $\lambda=-0.08$ is unstable, developing from a quasiperiodic trajectory (at $\lambda=-0.07$ ) to a periodic one (at $\lambda=-0.08$ ), to a tube orbit (at $\lambda=-0.085$ ) with a definitely chaotic motion (at $\lambda$ $=-0.09)$. This dramatic change in the $(2,18)$ torus is

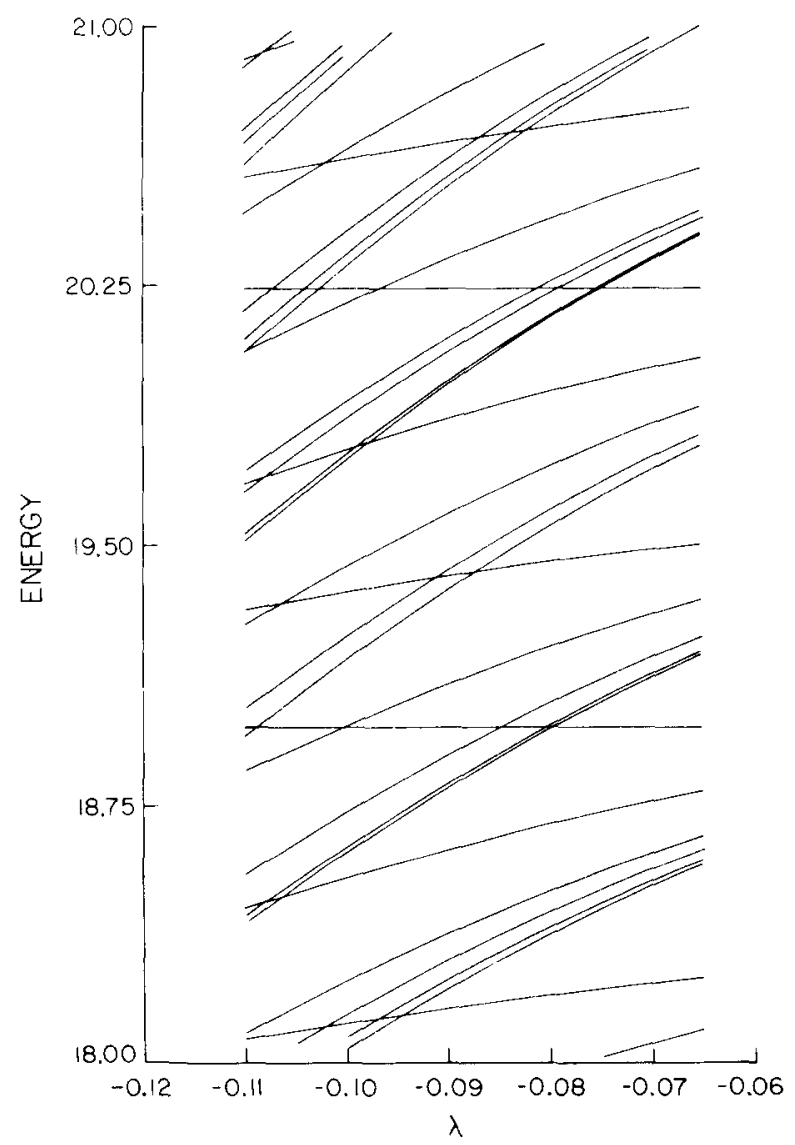

FIG. 1. Constant action curves for the action lattice with $\chi_{1}=\chi_{3}=-\frac{1}{2}$ and $c / 2 \pi=1$, for the Barbanis system. Note that the lowest multiple crossing around $\lambda=-0.08$ occurs at $E \sim 19.0$ units.

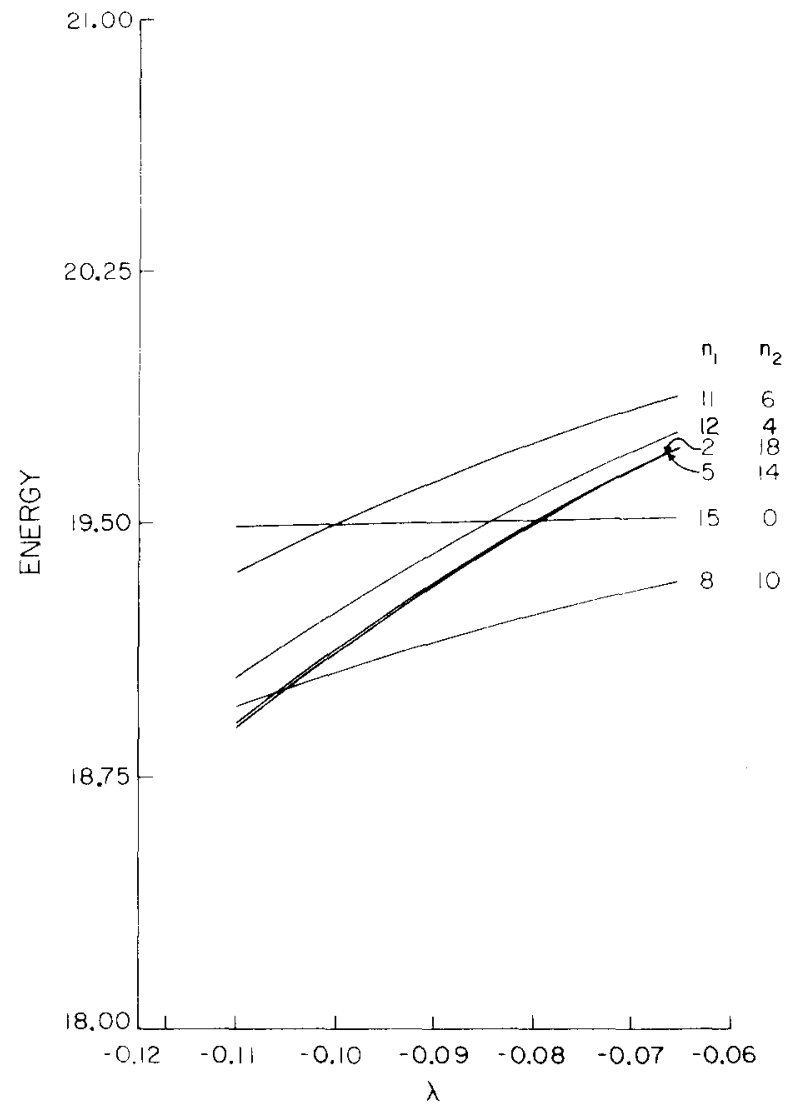

FIG. 2. Constant action curves for the lattice with $\chi_{1}=\chi_{2}=-\frac{1}{4}$ Only a few of the curves are shown here; the numbers $\left(n_{1}, n_{2}\right)$ are those in the actions $\left(n_{1}+\frac{1}{2}+\chi_{1}, n_{2}+\frac{1}{2}+\chi_{2}\right)$.

further evident in the Poincare surfaces of section shown in Fig. 4. We observe the change from a single smooth curve to a set of points, to a chain of 5 "islands" and finally to a more "random" surface of section. The ability of the present static description to sense the dynamics - the existence of stable and unstable fixed points that give rise to the island structure is clear. ${ }^{10}$ The $(5,14)$ torus undergoes a similar change. There is no such change in behavior of the $(11,6)$ torus; one can, however, see that the future avoided crossing with the $(15,0)$ torus is anticipated by the dimple in the surface of section; the avoided crossing there gives rise to two islands.

The associated spectra of the motion display the changes similarly, in Fig. 5. For the $(11,6)$ torus, the spectra at the different $\lambda$ values are virtually identical in their features, while for the $(2,18)$ torus, the spectrum changes from a discrete set of lines, to that characteristic of a chaotic trajectory.

An important consideration here is whether the chaotic trajectories individually sample all portions of the energy shell since they are no longer confined to tori. The results in Figs. 3 and 4 indicate that while these chaotic trajectories are not as restricted as the regular ones, they are nevertheless not ergodic on the energy shell; thus one cannot generally expect the average of any dynamical quantity along such a trajectory to very closely equal the microcanonical average at that energy. 

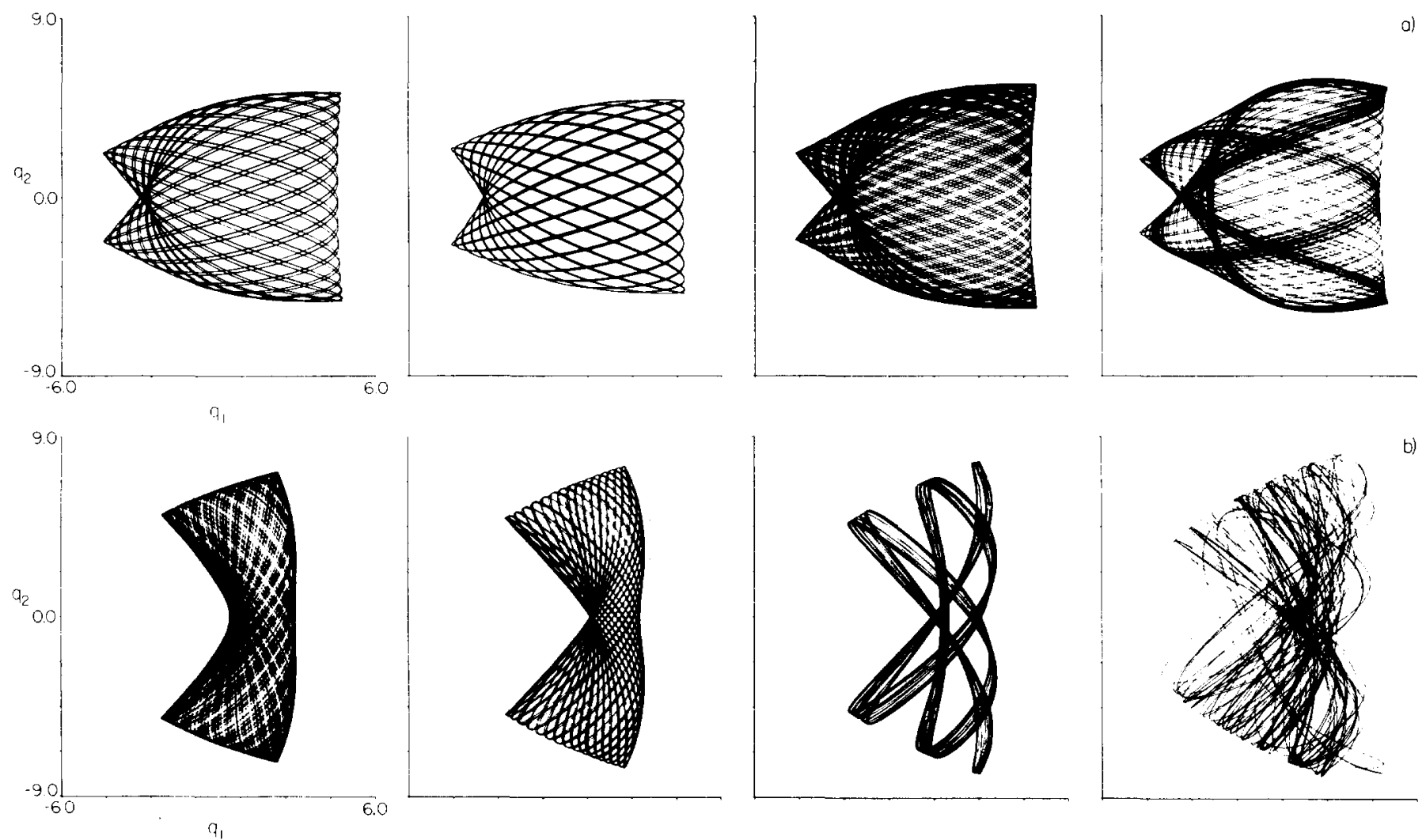

FIG. 3. Trajectories with fixed actions at different values of the parameter $\lambda$. From left to right, these are at $\lambda=-0.07, \lambda$ $=-0.08, \lambda=-0.085$, and $\lambda=-0.09$. The upper set of diagrams (a) are for the torus $(11,6)$, while the lower ones (b) are for the torus $(2,18)$. (The corresponding action curves are shown to Fig. 2.)
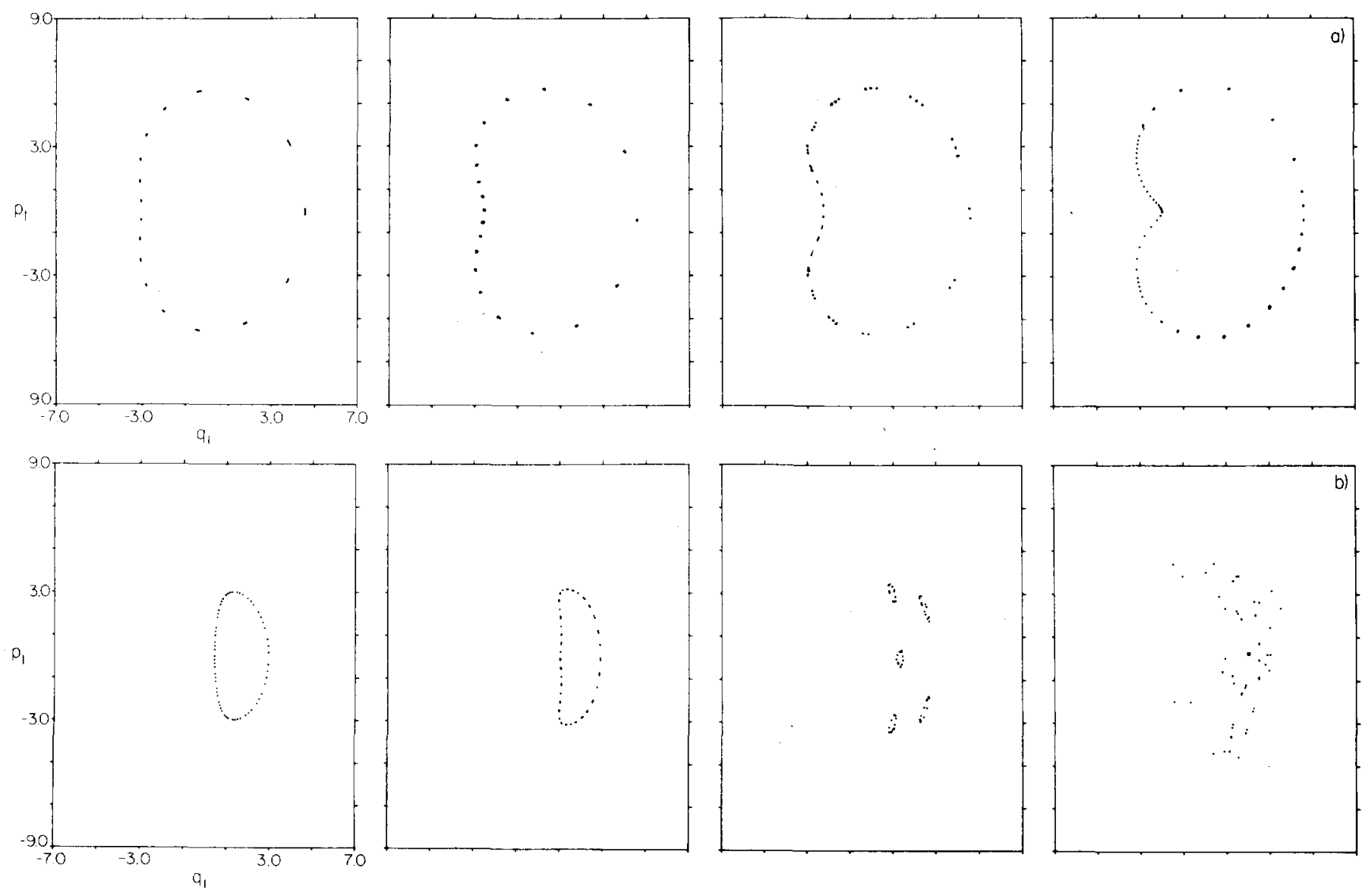

FIG. 4. The poincaré surfaces of section in the $\left(q_{2}=0, p_{2}>0\right) q_{1}-p_{1}$ plane, for the trajectories in Fig. 3. 

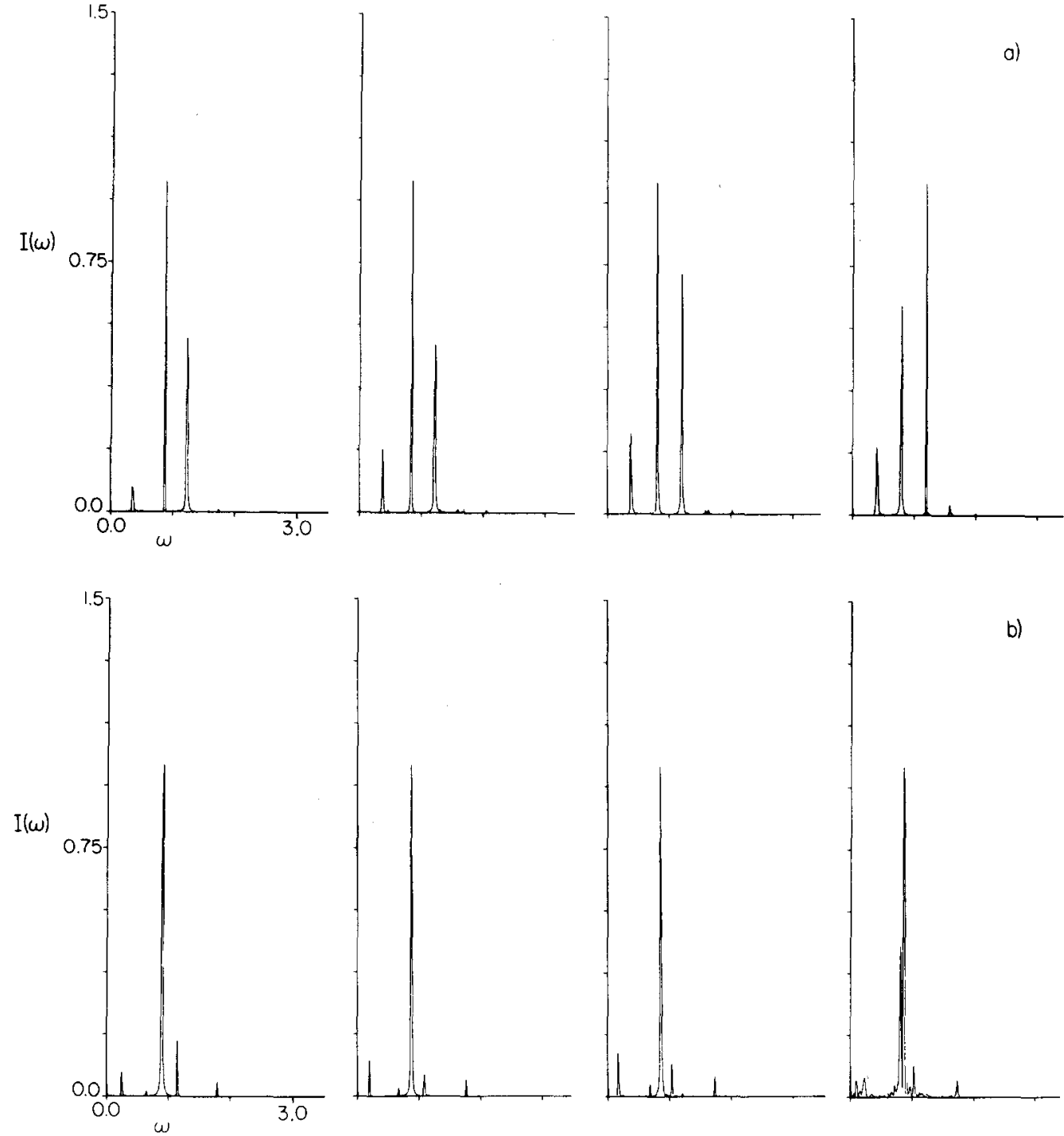

FIG. 5. The Fourier spectra of the trajectories in Fig. 3.

There has to be extensive chaos and mixing (perhaps extensive overlapping resonances in the Chirikov sense). 11 (b)

\section{B. Quartic perturbation}

In this section we apply the method to a somewhat different type of perturbing potential, which more clearly illustrates the effect of an isolated avoided crossing. This system is that of two coupled oscillators with a quartic perturbation, ${ }^{18}(0)$

$$
\mathfrak{C}=\frac{1}{2}\left(p_{1}^{2}+p_{2}^{2}+\omega_{1}^{2} q_{1}^{2}+\omega_{2}^{2} q_{2}^{2}\right)+\lambda q_{1}^{2} q_{2}^{2} .
$$

For the case $\omega_{1} \neq \omega_{2}$ to within $O(\lambda)$, the application of the Lie-transform technique is direct, and yields the normal form, which when expressed in terms of the actions $I_{1}$ and $I_{2}$, gives

$$
\begin{aligned}
K= & \omega_{1}\left(I_{1} / 2 \pi\right)+\omega_{2}\left(I_{2} / 2 \pi\right)+\lambda\left(I_{1} I_{2} / 4 \pi^{2} \omega_{1} \omega_{2}\right)-\frac{\lambda^{2}}{8 \pi^{3}} \\
& \times\left[\frac{\left(3 \omega_{2}^{2}-2 \omega_{1}^{2}\right) I_{1}^{2} I_{2}}{4\left(\omega_{2}^{2}-\omega_{1}^{2}\right) \omega_{2}^{3} \omega_{1}^{2}}+\frac{\left(2 \omega_{2}^{2}-3 \omega_{1}^{2}\right) I_{1} I_{2}^{2}}{4\left(\omega_{2}^{2}-\omega_{1}^{2}\right) \omega_{2}^{2} \omega_{1}^{3}}\right]+O\left(\lambda^{3}\right),
\end{aligned}
$$

which leads to the energy expression

$$
\begin{aligned}
E(\mathbf{n} ; \chi ; \lambda)= & \omega_{1}\left(n_{1}+\alpha_{1} / 4+\chi_{1}\right) c / 2 \pi+\omega_{2}\left(n_{2}+\alpha_{2} / 4+\chi_{2}\right) c / 2 \pi \\
& +\lambda / \omega_{1} \omega_{2}\left(n_{1}+\alpha_{1} / 4+\chi_{1}\right) c / 2 \pi\left(n_{2}+\alpha_{2} / 4+\chi_{2}\right) c / 2 \pi \\
& -\beta_{1}\left(n_{1}+\alpha_{1} / 4+\chi_{1}\right)^{2}\left(n_{2}+\alpha_{2} / 4+\chi_{2}\right) c^{3} /(2 \pi)^{3} \\
& -\beta_{2}\left(n_{1}+\alpha_{1} / 4+\chi_{1}\right)\left(n_{2}+\alpha_{2} / 4+\chi_{2}\right)^{2} c^{3} /(2 \pi)^{3}+O\left(\lambda^{3}\right)
\end{aligned}
$$

with

$$
\beta_{1}=\frac{-\lambda^{2}\left(3 \omega_{2}^{2}-2 \omega_{1}^{2}\right)}{4\left(\omega_{2}^{2}-\omega_{1}^{2}\right) \omega_{1}^{2} \omega_{2}^{3}} \quad \beta_{2}=\frac{-\lambda^{2}\left(2 \omega_{2}^{2}-3 \omega_{1}^{2}\right)}{4\left(\omega_{2}^{2}-\omega_{1}^{2}\right) \omega_{1}^{3} \omega_{2}^{2}} .
$$

Again, the Maslov indices $\alpha_{1}, \alpha_{2}$ are both 2. We choose the parameters $\omega_{1}^{2}=1, \omega_{2}^{2}=\frac{1}{2}$ and examine the system for a grid with $c=2 \pi, \chi_{1}, \chi_{2}=-\frac{1}{2}$ and in the vicinity of $\lambda$ $=-0.0125$. At this $\lambda$, the system dissociates at $E$ $=10.0$ units. Shown in Fig. 6 are the curves for the above action lattice, which gives a first set of overlapping avoided crossings at $\sim E=8.5$ units and thus the "chaotic transition" is predicted to occur at a somewhat higher energy relative to dissociation than in the previous example. The systematic study of a few tori in 


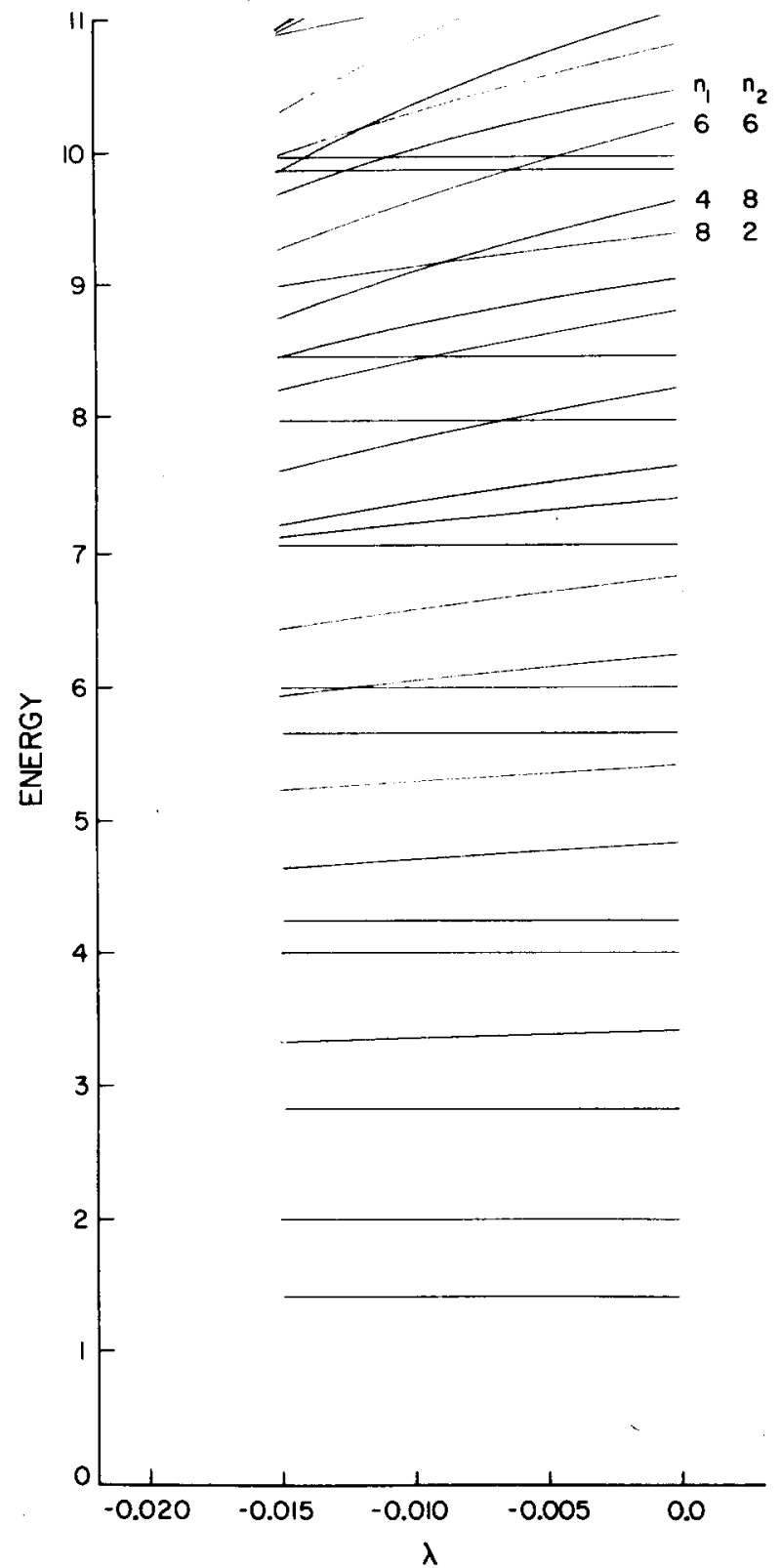

FIG. 6. Constant action curves for the quartic Hamiltonian, with $\chi_{1}=\chi_{2}=-\frac{1}{2}$ and $c / 2 \pi=1$. A few of the curves are labeled $\left(n_{1}, n_{2}\right)$; the tori corresponding to some of these curves are shown in Fig. 7.

this system is shown in Fig. 7. Of particular interest here is the effect of an isolated crossing which has been presumed to convert a torus to that of a periodic trajectory and, when the $E_{\lambda}$ vs $\lambda$ curve has passed through the crossing region, restore to the system its original torus. This behavior is dramatically seen in Fig. 7(b). A crossing occurred around $\lambda=-0.01$ for the tori with actions $(4,8)$ and $(8,2)$. Analogous changes were observed in the Poincare surfaces of section.

\section{Discussion}

For convenience we have chosen the unit of action, $c$ $=2 \pi$ in the foregoing examples. There is, however, a natural unit of action in these problems, related to the size of the secular perturbation terms, which cause actual crossings to become avoided crossings. The choice thus depends on the degree of detail desired. One might anticipate that a suitably normalized plot of number of crossings $N_{\lambda}$ in some interval $\Delta \lambda$ versus energy would be approximately independent of the choice of $c$, if there were enough crossings in the interval.

There is another point regarding the method presented here: The crossing of several action curves in some small $\lambda$ neighborhood is itself not sufficient to give rise to chaos, unless in addition, the "coupling" between such curves is enough to $\mathrm{mix}$ them, i.e., cause an overlap. Thus, in addition to information regarding the position of the crossings, one should additionally estimate the "width" of each avoided crossing in a more detailed examination. ${ }^{29}$

In the concluding part of this section we consider the relation between the present method of crossings to that of Chirikov. ${ }^{11(b)}$ When a crossing occurs here, for ac tions $\left(I_{1}^{\prime}, I_{2}^{\prime}\right)$ and $\left(I_{1}^{\prime \prime}, I_{2}^{\prime \prime}\right)$ one has

$$
K\left(I_{1}^{\prime}, I_{2}^{\prime}\right)=K\left(I_{1}^{\prime \prime}, I_{2}^{\prime \prime}\right)+O\left(\lambda^{p}\right),
$$

where $p=4$ and 2 in the examples given earlier. Expanding Eq. (3.8) yields, to order $\lambda^{p}$,

$$
\omega_{1}\left(I_{1}^{\prime}-I_{1}^{\prime \prime}\right)+\omega_{2}\left(I_{2}^{\prime}-I_{2}^{\prime \prime}\right)+O\left(\left[\mathbf{I}^{\prime}-\mathbf{I}^{\prime \prime}\right]^{2}\right)=0 .
$$

If $I_{1}^{\prime}-I_{1}^{\prime \prime}$ and $I_{2}^{\prime}-I_{2}^{\prime \prime}$ are sufficiently small, Eq. (3.9) reduces to Chirikov's condition, since we have chosen the grid of action with integer separations. Thus, within the approximation of neglecting the higher powers of $\mathbf{I}^{\prime}$, $I^{\prime \prime}$, the present method is useful for locating these internal resonances and the energy at which they occur, at a glance. Chirikov goes further and estimates the "widths" of these resonances; we intend to present an approximate method of doing this ${ }^{29}$ in a subsequent publication. For the exact trajectories, the width of the resonances would be related to the width, in action space, of the separatrix separating the islands such as those in Fig. 3(b).

If the higher order terms in Eq. (3.9) were truly negligible, then at the crossing of two curves one should have an exactly $\left(I_{1}^{\prime}-I_{1}^{\prime \prime}\right):\left(I_{2}^{\prime}-I_{2}^{\prime \prime}\right)$ resonant trajectory. Because of the approximations involved here, we usually locate only resonances that are close to this resonance, as in Fig. 7, where the two periodic trajectories have the ratio $\partial K / \partial I_{1}: \partial K / \partial I_{2}$ of $25: 33$ [Fig. $7(\mathrm{a})$ ] and $3: 5$ [Fig. $7(\mathrm{~b})]$ while the ratio $\left|I_{1}^{\prime}-I_{1}^{\prime \prime}\right|:\left|I_{2}^{\prime}-I_{2}^{\prime \prime}\right|$ is $(8-4)$ : $(8-2)$, i. e., $2: 3$.

\section{CONCLUSION}

Chaotic motion in dynamical systems is defined via the existence of orbital instability (cf. the discussion in Sec. I). However, the onset of such chaos is not a welldefined notion. ${ }^{10}$ We have shown in this paper, how one may detect the gradual destruction of toroidal motion by examining the energy dependence of tori with fixed actions, as a function of the perturbation. This enables one to locate in an approximate but nevertheless useful manner, which specific tori undergo destruction both in terms of total energy, as well as the region of phase space where such instability appears. In application to 

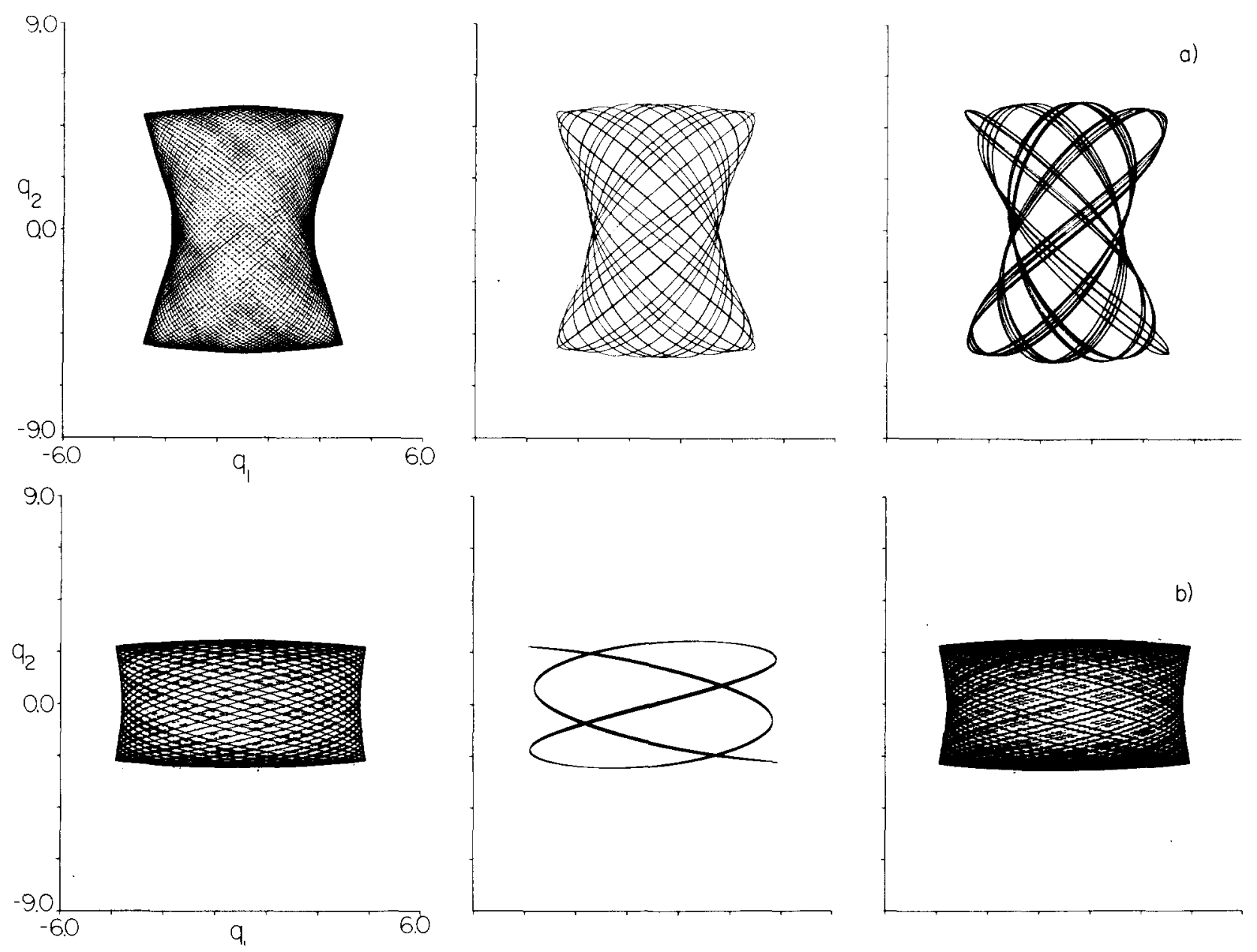

FIG. 7. Trajectories with fixed actions at different values of the parameter $\lambda$ for the Hamiltonian (3.5). From left to right, these are at $\lambda=-0.009, \lambda=0.010, \lambda=-0.011$, respectively. The upper set of diagrams, (a), are for the torus $(8,2)$, while the lower ones, (b) are for the torus $(4,8)$. The corresponding action curves are shown in Fig. 6 .

two simple two-degree-of -freedom systems, the utility of the method and the ease in its usage have been illus trated.

The interplay between classical and quantal ideas in this method of analysis leads to some interesting conse quences. Firstly, it follows from Sec. III, local classical chaos by no means implies classical ergodicity. Similarly, in the analogous quantum definition, ${ }^{13}$ the near degeneracy of several eigenstates (i.e., the quantum overlapping avoided crossings) probably does not give rise to quantum ergodicity (in the sense of average of dynamical quantities equalling the classical microcanonical ensemble average at that energy), but to a slightly restricted phenomenon. Extensive overlapping of many avoided crossings could however, approximate ergodicity. Secondly, while local classical chaos does not imply quantum chaos (in that the former can set in well before the latter), the actual workings of chaos are probably similar in many respects, especially in a semiclassical picture.

We have dealt with systems here that do not have zeroth order internal resonances. In systems with internal resonances, reduction to Birkhoff normal form is itself a trivial matter, ${ }^{30}$ but subsequent transformation to the $E$ polynomial requires explicit integration. ${ }^{18(b)}$ Thus the application of the method is straightforward, but requires more computation. This will be explored in future work. ${ }^{31}$

\section{ACKNOWLEDGMENTS}

We are pleased to acknowledge support of this research by a grant from the National Science Foundation. We have greatly benefited from conversations with Alan Weinstein.

${ }^{1}$ V. I. Arnol'd, Mathematical Methods of Classical Mechanics (Springer, New York, 1978); R. Abraham and J. E. Marsden, Foundations of Mechanics (Benjamin, New York, 1978).

${ }^{2}$ See, e.g., Nonlinear Dynamics, Conference Proceedings, New York Academy of Sciences, New York, 1980; Stochastic Behavior in Classical and Quantum Hamiltomian Systems, Volta Memorial Conference, Como 1977, edited by G. Casati and J. Ford, Springer Verlag Lecture Notes in Physics, 93 (1979).

${ }^{3}$ G. D. Birkhoff, Amer. Math. Monthly 49, 222 (1949).

${ }^{4}$ L. Markus and K. R. Meyer, Mem. Amer. Math. Soc. 144 (1974).

${ }^{5}$ (a) A. N. Kolmogrov, Dokl. Akad. Nauk, 98, 527 (1954); (b) V. I. Arnol'd, Usp. Mat. Nauk. 18, 13 (1963); (c) J. Moser, Nach. Akad. Wiss. Göttingen 1, 1 (1962). 
${ }^{6}$ See, e.g. , J. Ford in Fundamental Problems in Statistical Mechanics III, edited by E. D. G. Cohen (North-Holland, Amsterdam, 1975); also in Adv. Chem. Phys. 24, 155 (1973).

${ }^{7}$ M. Hénon and C. Heiles, Astron. J. 69, 73 (1964).

${ }^{8}$ D. W. Noid, M. L. Koszykowski, and R. A. Marcus, J. Chem. Phys. 67, 404 (1977); K. D. Hansel, Chem. Phys. 53, 35 (1978); I. C. Percival, J. Phys. A 12, 2053 (1979).

${ }^{9} \mathrm{G}$. Benettin, L. Galgani, and J. M. Strelcyn, Phys. Rev. A 14,2338 (1976).

${ }^{10}$ See, e.g., M. Tabor in Adv. Chem. Phys. (1980) for a recent review.

${ }^{11}$ (a) M. Toda, Phys. Letts. A 48, 335 (1974); P. Brumer and J. Duff, J. Chem. Phys. 85, 3566 (1976); C. Cerjan and W. Reinhardt, J. Chem. Phys. 71, 1819 (1979); (b) B. V. Chirikov, Phys. Repts. 52, 265 (1979); (c) J, M. Greene, J. Math. Phys. 20, 1183 (1979).

${ }^{12}$ R. Ramaswamy and R. A. Marcus, J. Chem. Phys. 74, 1379 (1981).

${ }^{13}$ R. A. Marcus in Nonlinear Dynamics (New York Academy of Sciences, New York, 1980); see also, D. W. Noid, M. L. Koszykowski, and R. A. Marcus, Chem. Phys. Lett. 73, 269 (1980).

${ }^{14}$ G. E. O. Giacaglia, Perturbation Methods in Nonlinear Systems (Springer, New York, 1972).

${ }^{15}$ G. D. Birkhoff, Acta Math. 43, 1 (1922); C. L. Siegel and J. K. Moser, Lectures on Celestial Mechanics (Springer, New York, 1972)

${ }^{16}$ I. C. Percival, J. Phys. B 6, 559 (1973); I. C. Percival, Adv. Chem. Phys. 36, 1 (1977).

${ }^{17}$ R. A. Marcus, Discuss Faraday Soc. 55, 34 (1973); a complete reference to the work of this group may be found in $D$. Noid, M. L. Koszykowski, and R. A. Marcus, J. Chem. Phys, 71, 2864 (1979).

${ }^{18}$ (a) S. Chapman, B. Garrett, and W. H. Miller, J. Chem.
Phys. 64, 502 (1976); (b) R. T. Swimm and J. B. Delos, J. Chem. Phys. 71, 1706 (1979); (c) I. C. Percival and N. Pomphrey, Mol. Phys. 31, 97 (1976); (d) C. Jaffe and W. P. Reinhardt, J. Chem. Phys. 71, 1862 (1979).

${ }^{19}$ A. Einstein, Verh. Deut. Phys. Ges. 19, 82 (1917); M. L. Brillouin, J. Phys. (Paris) 7, 353 (1926). J. B. Keller, Ann. Phys. 4, 180 (1958); V. Maslov, Theorie des Perturbations et Méthods Asymptotiques (Dunod, Paris, 1972).

${ }^{20} J$. J. Duistermaat, University of Utrecht, preprint (1980).

${ }^{21}$ (a) We have used the notation $(n+\alpha / 4+\chi)$ rather than $(n+\chi)$, but the latter could have been used instead. (b) While one could vary $x$ in the unit interval, such avoided crossings can be observed by looking at nearby $\lambda$ 's for fixed $\chi$.

${ }^{22} \mathrm{R}$. Burton and R. W. Easton, University of Colorado, preprint (1980).

${ }^{23}$ B. Barbanis, Astron. J. 71, 415 (1966).

${ }^{24} \mathrm{~K}$. Sorbie and N. C. Handy, Mol. Phys. 32, 1327 (1976).

${ }^{25}$ (a) G. I. Hori, Publ. Astr. Soc. Jpn. 18, 287 (1966). (b) G. I. Hori, Publ. Astr. Soc. Jpn. 19, 229 (1967).

${ }^{26}$ See the discussion in Sec. IIIC of the present paper.

${ }^{27}$ P. D. Siders, R. Ramaswamy, and R. A. Marcus (unpublished work). From a grid of values in $q_{1}, q_{2}$ and $\sin ^{-1}\left\{p_{1}[2(E-V)]^{-1 / 2}\right\}$ space, 25 pairs of trajectories were studied. Exponential separation was observed at $E=19,20$, and 21 in $2 / 24,6 / 24$ and $9 / 24$ of the cases respectively. On the energy shell, integration in phase space amounts to integration over three variables, $d q_{1} d q_{2}\left(d p_{1} / p_{2}\right)$ and we note that $d p_{1} / p_{2}$ equals $d\left(\sin ^{-1}\left\{p_{1}[2(E-V)]^{-1 / 2}\right\}\right)$.

${ }^{28}$ The notation here is $\left(n_{1}, n_{2}\right)$ to designate the actions $n_{1}+\frac{1}{2}+\chi_{1}$, $n_{2}+\frac{1}{2}+\chi_{2}$. For a given set of curves, $\chi_{1}$ and $\chi_{2}$ are fixed.

${ }^{29} \mathrm{An}$ example of a classical method is given in Ref. 13.

${ }^{30}$ F. G. Gustavson, Astron. J. 71, 670 (1966).

${ }^{31}$ P. D. Siders, R. Ramaswamy, and R. A. Marcus (in preparation). 